\title{
A Knowledge Discovery Methodology for Behavior Analysis of Large-Scale Applications on Parallel Architectures
}

\author{
Elias N. Houstis ${ }^{1,2}$, Vassilios S. Verykios ${ }^{1,3}$, Ann C. Catlin ${ }^{2}$, and John R. Rice ${ }^{2}$ \\ 1 Dept. of Computer and Commun. Engr., Univ. of Thessaly, Volos, GREECE \\ 2 Dept. of Computer Sciences, Purdue University, West Lafayette, IN, USA \\ 3 Research and Academic Computer Technology Institute, Patras, GREECE
}

\begin{abstract}
The focus of this paper is the application and extension of the knowledge discovery in databases process [5] developed in PYTHIA recommender system, to analyze the behavior of a DOE ASCI application/hardware pairs in the context of POEMS project[4]. The POEMS project has built a library of models for modeling scalable architectures like those in the ASCI program. Moreover, it supports detail simulation of a variety of state-of-the-art processors and memory hierarchies and incorporates parallel evaluation of discrete-event simulation. The driver application used is SWEEP3D.
\end{abstract}

Keywords: Recommender System, Data Mining, Knowledge Discovery, Scientific Computing

\section{Introduction}

One of the basic research issues in designing recommendation services is understanding the fundamental processes by which knowledge about scientific problems and their solutions is created. Some of this knowledge will come directly from experts - scientists and engineers - in the field. Other knowledge can be mined from experimental data arising from simulations. Yet, further knowledge will be learned from the experience gained by the recommendation service itself as it extracts performance knowledge about software components when these are running and applied to various problems.

The focus of this paper is the adoption of the knowledge discovery in databases process [5] of the PYTHIA recommender system, to the knowledge acquisition process for an ASCI application, in the context of the POEMS project [4]. PYTHIA $[7,10,8]$, a "scientific recommender system" whose users are scientists/engineers, is trying to locate software module(s) appropriate to their needs. Given a scientific problem instance and performance criteria constraints on its solution, PYTHIA recommends an algorithm, estimates its parameters, and identifies a location on the web where a software module implementing the algorithm

\footnotetext{
* This work was supported in parts by NSF grants, EIA-0103688, DMI-0122214, and EIA-0205663.
} 
can be obtained. The current implementations of PYTHIA address the domains of numerical quadrature, elliptic partial differential equations (PDEs), domain decomposition and linear algebra. PYTHIA's basis for recommendations is a rule-bank mined by spooling performance trace data for various algorithms on benchmark scientific problems and correlating variations in performance to specific characteristics of the problem input. In addition, PYTHIA interfaces with the GAMS software repository on the web, where implementations of the implemented algorithm can be downloaded. In this paper, we specifically concentrate on the data mining methodology utilized for the design of PYTHIA.

The POEMS project is building an initial library of models, at multiple levels of granularity, for modeling scalable architectures like those envisaged under the DOE ASCI program. The project supports evaluation of component behaviors through application of discrete-event simulation at multiple levels of detail. POEMS supports detailed simulation of a variety of state-of-the-art processors and memory hierarchies and incorporates parallel evaluation of discrete-event simulation. The project is also building a knowledge base from mining performance data that can be used to predict the performance properties of hardware/software as a function of their characteristics. POEMS development is being driven by modeling of the behavior of large-scale complex applications on parallel architectures. The first driver application is the SWEEP3D program that is being used to evaluate advanced parallel architectures at Los Alamos National Laboratories.

This paper gives a short introduction of the issues addressed in POEMS project and presents the design and implementation of the POEMS knowledge base (POEMSKB) in the context of SWEEP3D application and two computational environments associated with SP2 parallel machine and a cluster of Sun20 workstations. This paper is organized as follows. In Section 2 we describe the performance methodology we followed in POEMS and the case study used. In Section 3 we summarize the components of the POEMSKDD approach in terms of the components of the PYTHIA system and in Section 4 we present the results of the KDD process for POEMS. Conclusions are listed in Section 5.

\section{POEMS Performance Methodology and Sweep3D Case Study}

The major elements of the conceptual framework of POEMS are a general model of parallel computation and the use of associative objects as the representation basis for components. The models developed in POEMS span three different domains: application, operating system, and hardware. The application domain represents parallel computation as a dynamic task graph where nodes represent sequential computation units and edges represent dependencies. The operating system domain provides the models for process and memory management, interprocess communication, and parallel file systems. The hardware domain provides models for the processor and memory components, where the latter includes models from cache memory as well as shared memory hierarchies. 
As a proof of concept, the following performance analysis tools currently are being integrated in POEMS: an automatic task graph generator [1], the LogGP [9] and LoPC [6] analytic models, the MPI-SIM simulator [2], and the SimpleScalar instruction level processor/memory architecture [3]. Each tool contains capabilities for modeling the application domain, software domain, runtime system domain, and hardware domain. Together these tools provide the capability to analyze the performance of current and future applications and architectures, for very large and complex configurations and with a fairly high degree of confidence.

\subsection{POEMS Driver Application: SWEEP3D}

We consider the problem of predicting the performance of an ASCI kernel application called SWEEP3D on large scale parallel architectures such as IBM SP /2 or the SGI Origin2000. The benchmark code SWEEP3D represents the heart of a real ASCI application. It solves a 1-group time-independent discrete ordinates (Sn) 3D Cartesian (XYZ) geometry neutron transport problem. The XYZ geometry is represented by an IJK logically rectangular grid of cells. The angular dependence is handled by discrete angles with a spherical harmonics treatment for the scattering source. The solution involves two steps: the streaming operator is solved by sweeps for each angle and the scattering operator is solved iteratively.

This version of SWEEP3D is based on blocks of IJK and angles with a stride-1 line-recursion in the I-direction as the innermost work unit. The stride$1 \mathrm{I}$-line recursion is good for microprocessors and the blocked domains provide parallelism opportunities.

\subsection{POEMS Performance Database}

The POEMS performance database is to provide for the storage, retrieval and analysis of performance data, actual or estimated, for an application like SWEEP3D. The data includes: (a) actual measurements of performance, (b) data derived from actual measurements, e.g., a speedup curve that fits actual data, (c) simulation data derived from the "execution" of a low level simulator of the hardware and software environment, (d) specification data (for unavailable components of hardware or software), and (e) data derived from an analytical model, e.g., LogGP.

Currently the performance database has been modeled based on the underlying schema of the PYTHIA design. Following we present an instance of the PERFDATA table because of its major importance in the POEMS context. The PERFDATA record is shown in Figure 1. Some comments are in order. The record includes an id (name) and the system that is used for generating the performance data (PELLPACK). The database that this record correspond to is also listed (sweep3d), along with the name of the COMPOSITE record that includes the experiment that generated the data. The perfind_set attribute states the name of the file to be used that contain information related with the 


\begin{tabular}{|c|c|c|}
\hline name & I & sweep3d $50 \times 50 \times 50$ experiment SP2-6 \\
\hline system & I & pellpack \\
\hline comp_db & I & sweep3d \\
\hline composite_id & 1 & sweep3d 50x50x50 experiment \\
\hline perfind_set & 1 & sweep3d-std-par-grd \\
\hline solverseq & 1 & $50 \times 50 \times 501 \times 2$ sweep $3 \mathrm{~d} \mathrm{mk}=1, \mathrm{mmi}=1$ \\
\hline rundata & 1 & IBM SP2 with 18 compute nodes \\
\hline featurenames & I & \{"matrix symmetric","problem type"\} \\
\hline featurevals & I & $\{$ "no", "FEM" $\}$ \\
\hline nproc & 1 & 2 \\
\hline CSwitch & 1 & $\{8715,8638\}$ \\
\hline SysCalls & I & $\{72886,72790\}$ \\
\hline PgFlts & 1 & $\{2224,2215\}$ \\
\hline PktsRd & 1 & $\{294,247\}$ \\
\hline PktsSt & I & $\{186,173\}$ \\
\hline mean_UserStat & 1 & $\{98.82,98.98\}$ \\
\hline mean_KernelStat & 1 & $\{0.69,0.53\}$ \\
\hline mean_WaitStat & 1 & $\{0.11,0.06\}$ \\
\hline mean_IdleStat & 1 & $\{0.38,0.43\}$ \\
\hline std_UserStat & I & $\{6.92,6.87\}$ \\
\hline std_KernelStat & 1 & $\{2.61,2.59\}$ \\
\hline std_WaitStat & 1 & $\{2.54,1.45\}$ \\
\hline std_IdleStat & I & $\{5.21,5.76\}$ \\
\hline SENDing & 1 & $\{1.1922,0.748\}$ \\
\hline RECVing & 1 & $\{86.891,5.4036\}$ \\
\hline BCASTing & I & $\{0.0008,0.1129\}$ \\
\hline BARRIER & I & $\{0.0005,0.0722\}$ \\
\hline ALLREDUCINGing & I & $\{0.0005,0.0722\}$ \\
\hline perfproc2names & 1 & \{"BytesTo", "SendsTo", "BytesFrom" , "RecvsFrom"\} \\
\hline \multirow[t]{2}{*}{ perfproc2vals } & 1 & $\{\{\{" 5760000 ", " 14400 "$, "5760000" , "5760000" $\}\}$, \\
\hline & I & $\{\{" 5760000 ", " 14400 "$, "5760000", "5760000" $\}\}\}$ \\
\hline modnames & I & \{"domain processor", "decomposer", "triple"\} \\
\hline timeslice & 1 & \{"elapsed", "communication"\} \\
\hline \multirow[t]{2}{*}{ time } & 1 & $\{\{\{" 0 ", " 0 "\},\{" 0 "$, "0" $\},\{" 302.1300049 ", " 2.7499979 "\}\}$, \\
\hline & l & $\{\{" 0 "$, "0" $\},\{" 0 "$, "0" $\},\{" 301.7099915 ", " 2.7699978 "\}\}\}$ \\
\hline total & l & $\{" 302.1300049 "$, ,301.7099915"\} \\
\hline
\end{tabular}

Fig. 1. PERFDATA record for a 2-processor configuration in SP2.

data collection process. The particular sequence from the COMPOSITE record that generated the data is also referenced along with the RUNDATA record. In some cases, there are features that are dependent neither upon the application nor upon the underlying machine. These features are mostly related with the specific run, and for this reason have been included in the PERFDATA record such as the ones included as values for the attributes featurenames and featurevals. As we said, this record correspond to a parallel run of the SP2 
system. For this reason, the number of processors of the parallel machine that the data was collected from is also explicitly stated in the record. In addition to the above information, there are four groups of metrics whose values are kept in the PERFDATA record. The first group corresponds to information related to the processes running in each processor and other system and user specific information. The second group of measurements include information related to the number of bytes communicated between every pair of processors. Actually, the corresponding field is a three dimensional array that states the $i d s$ of the processors communicated along with the number of bytes exchanged in one run of the application. The third group of measurements include timing information related to the various modules of the PELLPACK system. The last group include information related to the total timing of the application in per processor basis. Although PELLPACK allows us to extract timing information for each module, it does not provide us with the capability to take measurements related to the performance behavior of the underlying system. For this reason, we have incorporated into PYTHIA, various profilers that provide us with trace data from different platforms.

\section{Components of POEMSKDD}

The implementation of the POEMSKDD follows closely the design of PYTHIA system. The system consists of all the layers included in the architectural diagram of PYTHIA system with the only difference that the statistical analysis module performs a slightly different operation than simply ranking of system modules.

For the POEMSKDD we have made use of a well known clustering tool that partitions the space of the performance data independently of its dimension. For the interpretation of the actual meaning of the identified clusters, we use the decision tree inducer from the pattern extraction module of PYTHIA. After the classes have been described, the decision tree inducer runs once again in order to build a classification model to be used for prediction purposes. At this time, the inducer takes as an input the parameters of the machine/application pair along with the label assigned to each pair by the clustering tool for building a model that predicts the performance class of a certain combination of application/machine parameters. Following, we give a detailed description of the clustering phase for the SWEEP3D raw performance data.

\subsection{Clustering of SWEEP3D Performance Data}

The information we have collected for the SWEEP3D application, includes application and machine feature data as well as performance data from running the application in an SP/2 parallel machine. In particular we make use of the grid size for the three dimensions, $x, y$ and $z$, the parameter $m k$ ( $K$-planes for blocking), and $m m i$ (the angles for blocking), the number of processors used for executing the application, as well as measurements for the elapsed and communication time. Thus, we have generated a feature vector for our study of size 8 . We 
have only used this small number of variables for demonstration purposes and also because this set adequately reflects the variation in the underlying domain for observing the interesting relationships.

The first step in discovering knowledge from the collected data is to consider what kind of task must be applied in this case study. The main problem is that in such an environment where we are looking for patterns without having any a-priori knowledge of the trends that exist in them, we need to start the data analysis by identifying subsets of data with similar characteristics or else clusters. This is what is usually called as clustering. Clustering, is a common descriptive task where one seeks to identify a finite set of categories or clusters to describe the data. For partitioning the performance data space into clusters in this study we have made use of the AutoClass system, a well-known clustering tool developed by NASA Ames Research Center.

The classes we are after in this knowledge extraction engagement are related to the performance of the underlying parallel machine for the various application parameters. For this reason, the cluster generation process is given as input only the performance information that is kept in the feature vector mentioned before. This information include the elapsed and communication time spent for executing the application in the parallel machine. The model we have selected for both variables is a Multi Normal model which implements a likelihood term representing a set of real valued attributes, each with constant measurement error and without missing values, which are conditionally independent of other attributes given the class. This is an assumption that we make and is related to the specific nature of the performance data we use. A pictorial representation of classes in the 2D space of performance data is given in Figure 2. The clustering tool identified 6 classes. The visualization tool has re-mapped the identified classes like follows: 4 to 1,0 to 2,1 to 3,2 to 4,3 to 5 , and 5 to 6 .

The performance data along with the corresponding classes identified by AutoClass are provided to a classification tool for the description of the performance classes. We have once again used the ID3 software and the decision tree produced is presented in Figure 3.

\subsection{Feature-Based Cluster Description}

After the identification and description of performance classes, we need to associate them with application/machine features, in order to be able to decide or predict the performance class of a certain pair of application and machine configuration based on their static characteristics without having to run the application. This is not only very efficient, but sometimes it is the only feasible way. For example, in this manner we can investigate how the number of processors relates to the performance of the system, and based upon the results, we might decide to change the configuration of the system.

For this step, we use four features. The first feature is the problem size, which is the product of the 3 grid sizes in the 3 dimensions, the second is the number of processors (nproc) used for running the application, and the last two the $m k$ and $m m i$ parameters that are related closely to the application. We consider two 


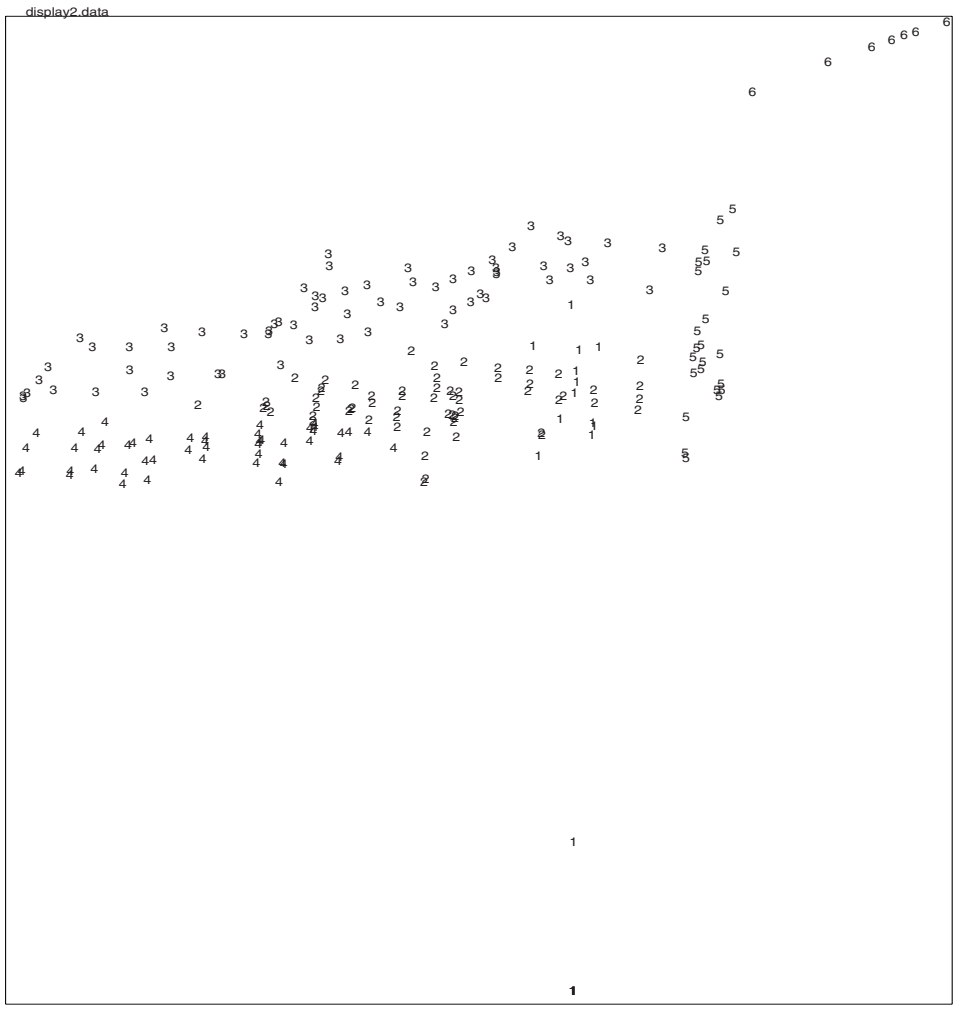

Fig. 2. Pictorial representation of the distribution of classes in the $2 \mathrm{D}$ space. The values of the elapsed time are depicted in the $x$ axis and the values of the communication time are depicted in $y$ axis.

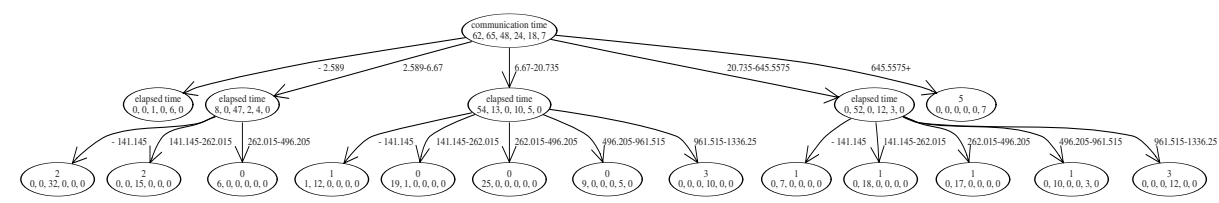

Fig. 3. Decision tree generated for the description of the performance classes identified by AutoClass.

of these variables, the number of processors and the problem size as continuous ones, while we consider the other two as categorical, because they only take values from a very small set of integers. The result of this classification task by using ID3 can be observed in Figure 4. 


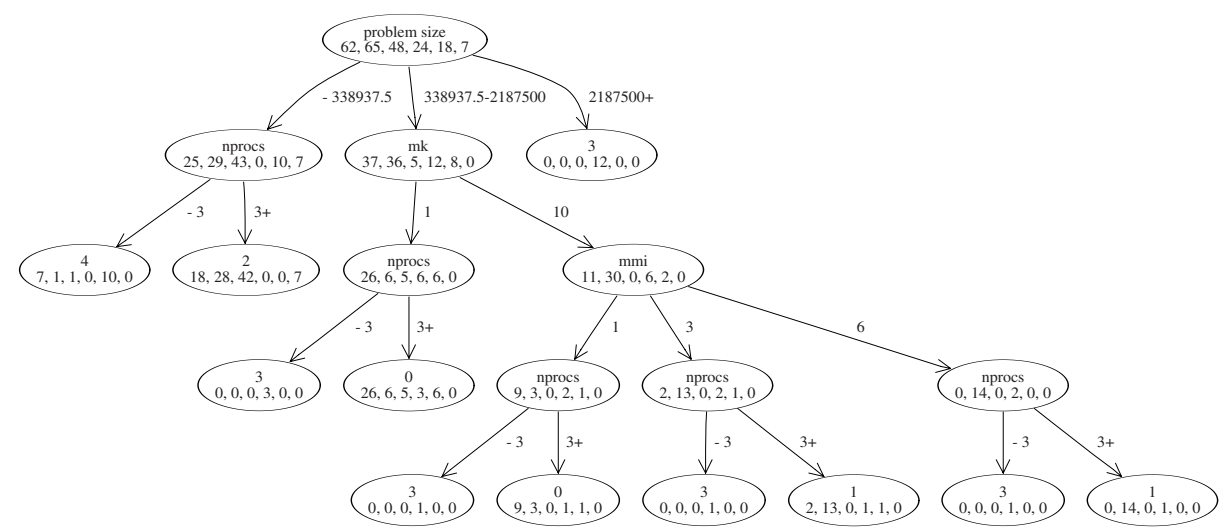

Fig. 4. Decision tree for identifying the performance classes based on static application and machine characteristics.

\section{The Results of the KDD Process on SWEEP3D Performance Data}

The decision tree corresponding to the description of the performance classes identified by AutoClass and shown in Figure 3 attains strong classification patterns, in the sense that the condition vector (ordered set of values shown inside a lead node) for the leaf nodes, contains cases/examples that belong to only one class. For example, we can state that the class 2 is very efficient with respect to both the communication and elapsed time.

The decision tree generated for predicting the performance classes based on static application and machine characteristics shown in Figure 4 does not deliver strong classification because more than one set of examples is covered by most of the leaves in the induced tree. This is an indication that the decision tree algorithm did not have enough features for explaining the data. This does not create any problem though for deriving rules that the recommender system can use for predicting the performance of a machine with some specific parameter values.

- A general observation that can be made from the data is that even for the minimum problem size of applications which were tried in these experiments, and the maximum number of processors, the application does not exhibit excessive communication volume, which means that the application scales really well for the problems sizes and number of processors considered in this case study.

- Another result which can be derived from the patterns in the decision tree (Figure 4) is that there is an ordering of the parameters used for explaining 
the performance behavior. This ordering implies that the problem size has the highest discriminating power for separating the data into performance classes, the parameter $m k$ has the second highest, the parameter $m m i$ has the third highest and the number of processors has the lowest discriminating power.

- Classes 0 and 1 exhibit almost similar performance behavior with only difference that class 1 exhibits higher communication times than class 0 . Both classes exhibit medium to high communication times and small to large elapsed times. This behavior can be attributed mainly to the large number of processors.

- Class 2 exhibits small communication and elapsed time. The large majority of the examples are characterized either by small problem size, and a large number of processors or medium problem size, or by medium problem size, $m k=1$ and large number of processors.

- Class 3 exhibits medium to high communication time and a very high elapsed time. Half of the examples belonging to this class are characterized by large problem sizes. The other half is distributed equally between $m k=1$ and $m k=10$. For those having $m k=10$, the examples are furthermore equally distributed among all the possible values of the parameter mmi. Notice that for all the previous cases the examples are equally distributed as well between the two categories for the number of processors.

- Class 4 exhibits very stable medium elapsed time but it spans a big range of communication times from small to medium-high ones. The examples falling under this class are characterized either by small problem size and small number of processors, or medium problem size and large number of processors.

- Class 5 exhibits high communication time. All the examples covered by class 5 are characterized by small problem size and large number of processors. This fact completely explains the high communication volume.

\section{Conclusions}

In this paper we have extended the KDD methodology of the PYTHIA system to address the selection of software/machine pairs and their parameters in the context of an environment for designing high performance application/machine pairs and predicting its performance. In this approach, the designer can utilize different performance models at the application, architecture, and OS levels. The goal of the KDD methodology in this paper is to identify various relationships of the system parameters involved with the application parameters. For example, the system can predict the optimum number of processors for a given machine out of many application partitionings or the best partitioning of the application with respect to various levels of grid sizes. PYTHIA has been extended to make these predictions. The PYTHIA database schema is adequate for accommodating the needs of various modeling requirements. 


\section{References}

1. V. S. Adve and R. Sakellariou. Application Representations for Multi-Paradigm Performance Modeling. International Journal of High Performance and Scientific Applications, 1999. To appear.

2. R. Bagrodia and S. Prakash. Using Parallel Simulation to Evaluate MPI Programs. In Proceedings of the 1998 Winter Simulation Conference WSC '98, 1998.

3. Doug Burger and Todd M. Austin. The SimpleScalar Tool Set, Version 2.0. Technical Report Technical Report \#1342, Computer Sciences Department, University of Wisconsin-Madison, June 1997.

4. E. Deelman, R. Bagrodia, A. Dube, J. Browne, A. Hoisie, Y. Luo, O. Lubeck, H. Wasserman, R. Oliver, R. Teller, D. Sundram-Stukel, M. Vernon, V. Adve, E. Houstis, and J. Rice. POEMS: End-to-end Performance Design of Large Parallel Adaptive Computational Systems. In Proceedings of the 1st International Workshop on Software and Performance (WOSP) '98, October 1998.

5. U. M. Fayyad, G. Piatetsky-Shapiro, and P. Smyth. From Data Mining to Knowledge Discovery: An Overview. In U. M. Fayyad, G. Piatetsky-Shapiro, P. Smyth, and R. Uthurusamy, editors, Advances in Knowledge Discovery and Data Mining, pages 1-34. AAAI Press/MIT Press, 1996.

6. M. I. Frank, A. Agarwal, and M. K. Vernon. LoPC: Modeling Contention in Parallel Algorithms. In Proceedings of the 6th ACM SIGPLAN Symposium on Principles and Practices of Parallel Programming (PPoPP '97), 1997. Las Vegas.

7. E. N. Houstis, S. Weerawarana, A. Joshi, and J. R. Rice. The PYTHIA Project. In S. K. Aityan, editor, Neural, Parallel, and Scientific Computations, pages 215-218. Dynamic Publishers., 1995.

8. Elias N. Houstis, Ann C. Catlin, John R. Rice, Vassilios S. Verykios, Naren Ramakrishnan, and Catherine E. Houstis. PYTHIA-II: A Knowledge/Database System for Managing Performance Data and Recommending Scientific Software. ACM Transactions on Mathematical Software, 26(2):227-253, 2000.

9. A. M. Ionescu, K. E. Schauser, and C. Scheiman. LogGP: Incorporating Long Messages into the LogP Model. In Proceedings of the 7th Annual ACM Symposium On Parallel Algorithms and Architectures (SPAA '95), 1995. Santa Barbara, July.

10. S. Weerawarana, E. N. Houstis, J. R. Rice, A. Joshi, and C. E. Houstis. PYTHIA: A Knowledge Based System to Select Scientific Algorithms. ACM Transactions on Mathematical Software, 22(4):447-468, 1996. 